EPJ Web of Conferences 59, 02006 (2013)

DOI: $10.1051 /$ epjconf/20135902006

(C) Owned by the authors, published by EDP Sciences, 2013

\title{
Study of shock-coalescence on the LIL laser facility
}

G. Debras ${ }^{1, a}$, C. Courtois ${ }^{1}$, F. Lambert ${ }^{1}$, S. Brygoo ${ }^{1}$, A. Duval $^{1}$, S. Darbon ${ }^{1}$, B. Villette ${ }^{1}$, I. Masclet-Gobin ${ }^{1}$, F. Philippe ${ }^{1}$, A. Casner ${ }^{1}$, P. Seytor ${ }^{1}$, L. Videau ${ }^{1}$, H. Graillot ${ }^{2}$, T. Chies ${ }^{2}$, O. Henry², D. Raffestin ${ }^{2}$ and C. Chicanne ${ }^{3}$

${ }^{1}$ CEA, DAM, DIF, 91297 Arpajon, France

${ }^{2}$ CEA, DAM, CESTA, 33114 Le Barp, France

${ }^{3}$ CEA, DAM, VALDUC, 21120 Is-sur-Tille, France

\begin{abstract}
We use the LIL (Ligne d'Intégration Laser) facility to study the coalescence of two planar shocks in an indirectly-driven planar sample of polystyrene. This experiment represents the preliminary stage of the future shock-timing campaign for the Laser Megajoule (LMJ). The main objectives are to validate the experimental concept and to test the numerical simulations. We used a gold spherical hohlraum to convert into X-ray the $351 \mathrm{~nm}$ wavelength laser pulse and to initiate the two shocks in the sample. To access time resolved shock velocities and temperature, we used two rear-side diagnostics: a VISAR (Velocity Interferometer System for Any Reflection) working at two different wavelengths and a streaked optical selfemission diagnostic. We observed the coalesced shock, in good agreement with the numerical simulations. We also observed a loss of signal during the first nanoseconds probably due to sample heating from the hohlraum X-ray flux.
\end{abstract}

\section{CONTEXT}

The LMJ, like the National Ignition Facility (NIF) in the USA, is designed to achieve thermonuclear ignition in an indirect drive scheme, by imploding a spherical capsule filled with a deuterium-tritium (DT) mixture. The best way to efficiently compress the fuel is quasi-isentropic compression. To approximate this compression, a series of four successive centripetal shocks are sent into the sample [1]. The shocks need to be accurately controlled in timing and strength to coalesce at the same time in the gaseous DT close to the inner surface of the cryogenic DT. The first three shocks allow compressing the capsule quasi-isentropically, keeping the cryogenic DT on a low adiabat. The fourth shock drives the final implosion until the ignition. This scheme requires to adjust the laser power time history entering in the gold hohlraum.

In this experiment, we seeked to validate the experimental concept in planar geometry and indirect drive for the first two shocks using a truncated LMJ-like pulse shape and to compare the results with numerical simulations.

\section{EXPERIMENTAL SETUP}

For this experiment we used the LIL laser facility at $3 \omega(351 \mathrm{~nm})$ with an energy of $10 \mathrm{~kJ}$ in $12 \mathrm{~ns}$. A spherical gold hohlraum was used to obtain a radiation temperature similar to the first part of the nominal LMJ-drive. Two planar shocks are sent in a planar sample of polystyrene (PS $-\mathrm{C}_{8} \mathrm{H}_{8}$ ) fixed on

\footnotetext{
${ }^{a}$ e-mail: gregoire.debras@cea.fr
}

This is an Open Access article distributed under the terms of the Creative Commons Attribution License 2.0, which permits unrestricted use, distribution, and reproduction in any medium, provided the original work is properly cited. 

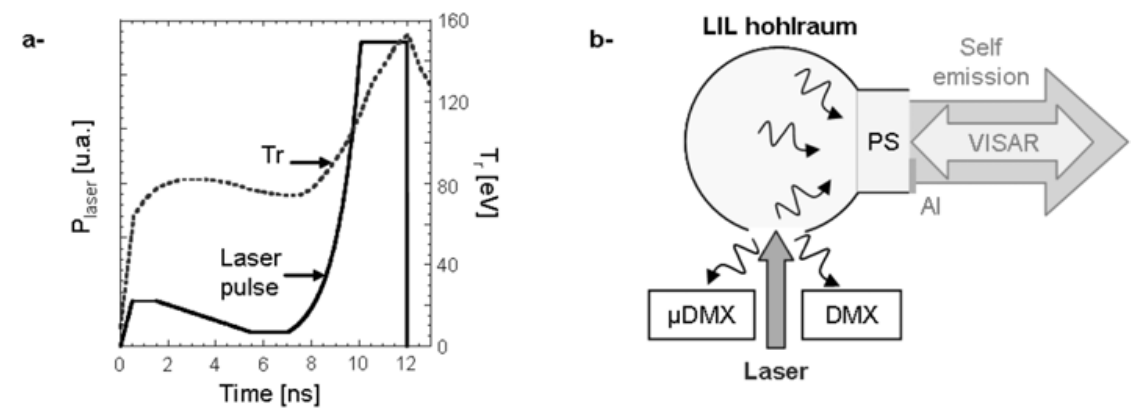

Figure 1. a) Profiles of the laser power and the associated radiation temperature in the nominal case ( $10 \mathrm{~kJ}$ in $12 \mathrm{~ns}$ at $3 \omega)$; b) experimental setup scheme.

the hohlraum at $90^{\circ}$ to the laser axes. We present in figure 1 the experimental setup, with the profiles of the laser power and radiation temperature in the nominal case.

We measured the radiation temperature of the hohlraum using two wide-band X-ray spectrometers ( $\mu \mathrm{DMX}$ and DMX) [2]. Two rear-side diagnostics measured the velocity and the self emission of the shock, respectively the VISAR [3, 4] and the streaked optical self emission diagnostic. The VISAR is composed of two channels working at two different wavelengths, $1 \omega(1064 \mathrm{~nm})$ and $2 \omega(532 \mathrm{~nm})$. This probe beam reflects at early time on the first shock, and when the second, faster, catches it, the probe beam reflects on the coalesced shock. The self emission is observed over a wavelength domain of $100 \mathrm{~nm}$, centered at $650 \mathrm{~nm}$.

The front side of the $500 \mu \mathrm{m}$ PS sample is coated with aluminium to determine the time origin corresponding to the first X-rays produced in the hohlraum. On the rear side, an aluminium shield masks a third of the target to get signal independently of shock properties.

Because PS blanking by the X-rays flux was expected, three types of laser pulse shapes have been studied to investigate: a simple case that produces only one shock from a truncated pulse shape at $7 \mathrm{~ns}$, a nominal case with $12 \mathrm{~ns}$ pulse, and a truncated pulse at $10 \mathrm{~ns}$ that produces two shocks with reduced heating effects.

\section{EXPERIMENTAL RESULTS AND COMPARISON WITH NUMERICAL SIMULATIONS}

The first shot we discuss used a $7 \mathrm{~ns}$ truncated pulse to initiate a single shock. The rear side diagnostics images are presented in figure 2 -a. We observe on the VISAR interferogram ( $1 \omega$ channel because of poor reflectivity on the $2 \omega$ channel) two parts in the space dimension, one corresponding to a reflection on the aluminium shield with straight fringes, the other associated to the reflection through the sample. After the initial time $t_{0}$ two regions are apparent: at first $\left(1^{*}\right)$ we lose the VISAR signal and see ghost fringes coming from reflection on the vacuum-PS interface. This extinction corresponds to PS preheating and lasts until the shock overtakes the opaque zone, as observed on one-dimensional radiation hydrodynamics simulation with the CEA code ESTHER [5]. The VISAR probe beam reflects on the shock $\left(2^{*}\right)$ and we observe the fringes moving proportionally to the shock velocity. We observe a decreasing shock velocity. The self emission also has the same characteristics with a loss of signal and then a decreasing signal consistent with a decreasing velocity.

The velocity profile of the shock obtained from the VISAR is compared to the numerical simulations (figure 2-b) from the 2D radiation hydrodynamics FCI2 code at CEA [6]; the two results are in good agreement within the error bars. The SESAME 7592 equation of state (EOS) table [7] was used for the sample. Using the same table, we inferred the shock temperature from its velocity and compared it to the temperature obtained from the self-emission calibrated measurement. The two temperature profiles are similar with a decrease from $2 \mathrm{eV}$ at $5 \mathrm{~ns}$ to $0.6 \mathrm{eV}$ at $25 \mathrm{~ns}$, which supports the choice of 

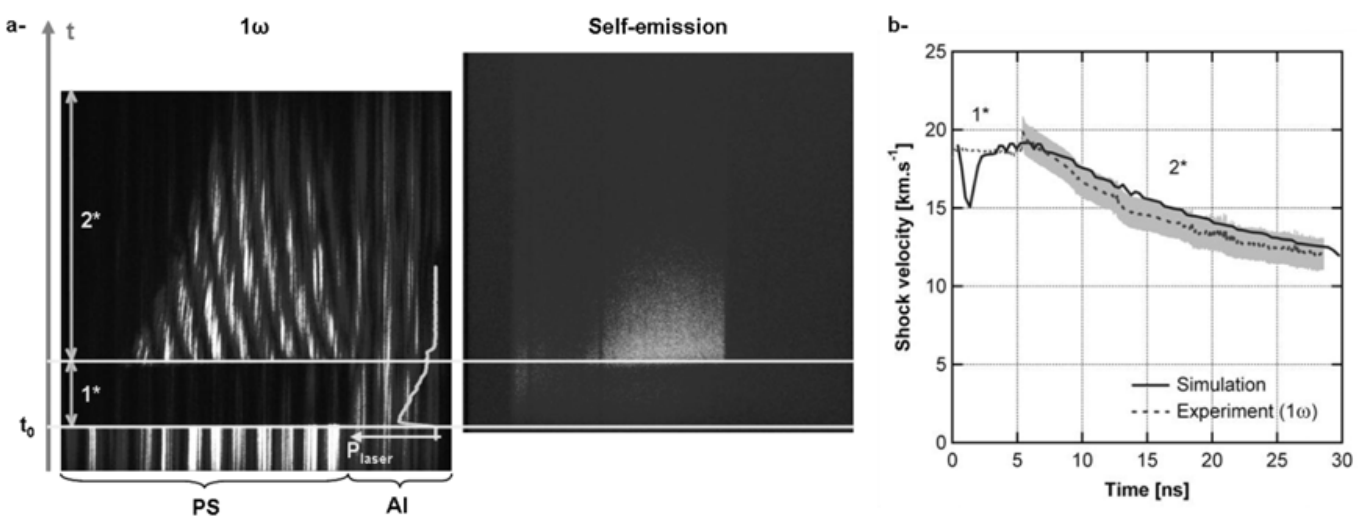

Figure 2. a) Images from the $1 \omega$ VISAR channel with the laser power profile (at left) and streaked self emission (at right) for a $7 \mathrm{~ns}$ pulse; b) Comparison between experimental and numerical simulations of the shock velocity profile.
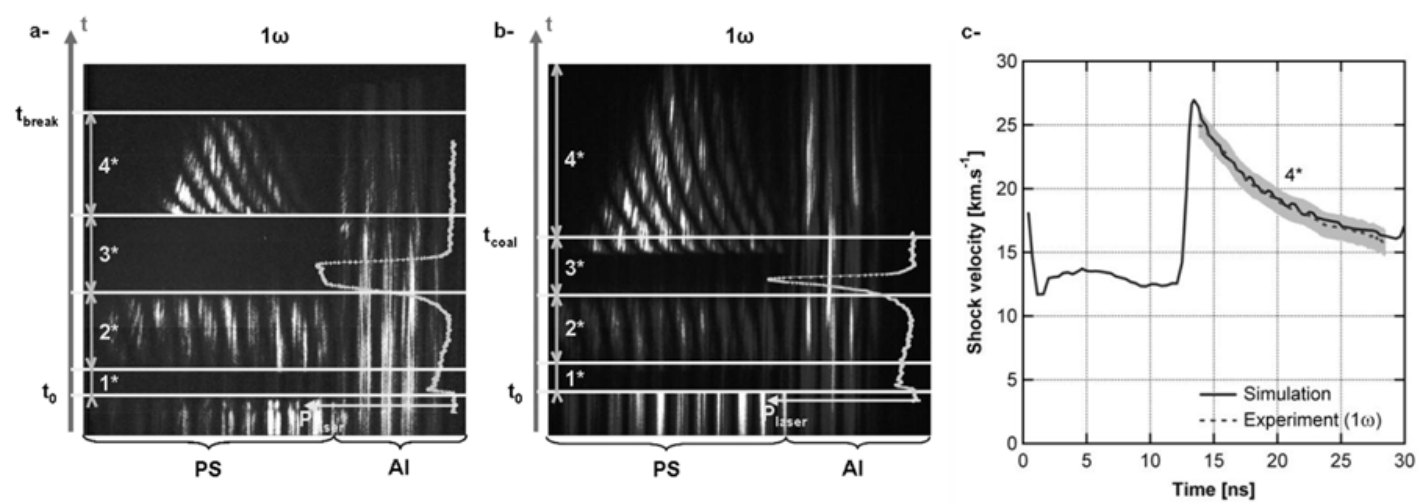

Figure 3. a) VISAR interferogram with the nominal laser power profile; b) idem for the $10 \mathrm{~ns}$ truncated case; c) comparison of the velocity profiles between experiment and simulation for the $10 \mathrm{~ns}$ case.

this EOS for the simulation. Other calculation with CH SESAME 7590 has shown us a discrepancy with experimental results.

The next shot presented is nominal with a 12 ns pulse. On the $1 \omega$ VISAR interferogram (figure 3-a) we distinguish the first two regions with preheating $\left(1^{*}\right)$ and the reflection on the first shock $\left(2^{*}\right)$, as in the single shock case but with a weaker shock because of the lower foot of the laser pulse. Two other regions appear, with a quasi-total blanking of the PS sample caused by a volume heating with the second laser peak $\left(3^{*}\right)$, associated to gold M-band, and the return of the signal that we identify as the reflection on the coalesced shock until the breakout $\left(4^{*}\right)$. We clearly observe the heating phenomenon which prevents to see the coalescence.

To limit the hard X-ray influence, we used a $10 \mathrm{~ns}$ truncated pulse, (figure 3-b). We observe the same four regions. The blanking region $\left(3^{*}\right)$ is less important and the coalescence appears with a fringe displacement between $\left(3^{*}\right)$ and $\left(4^{*}\right)$. Just before the coalescence the probe beam is reflected on a surface which is probably not the first shock, its reflectivity is not consistent with its velocity. We plot on figure 3-c the shock velocity profile from the $10 \mathrm{~ns}$ VISAR results, which are in good agreement with numerical simulations. 

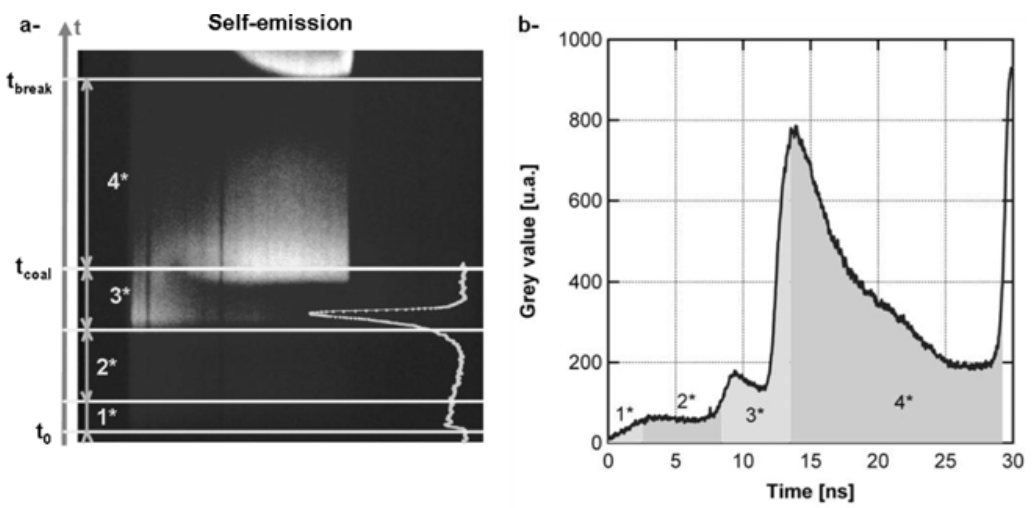

Figure 4. a) Streaked self-emission image with the laser power profile (10 ns); b) averaged time profile determined from the self-emission image.

For the $10 \mathrm{~ns}$ case we present in figure 4-a and 4-b respectively the image of the self-emission diagnostic and a spatially average time history. We observe different emission peaks followed by a signal decrease, which are consistent with the VISAR probe beam reflectivity. We can see the end of preheating between $\left(1^{*}\right)$ and $\left(2^{*}\right)$. The emission peak in $\left(3^{*}\right)$ corresponds probably to light coming directly from the second laser peak and not from the sample itself. The peak at the beginning of $\left(4^{*}\right)$ corresponds to the coalescence. A sharp increase of the self emission is observed just at the shock breakout.

\section{CONCLUSIONS}

The experiment has validated the experimental concept. We have proved we can produce and observe the coalescence of two shocks in indirect drive on the LIL laser facility. We can determine the shocks dynamics with the rear side diagnostics and access information about PS under X-ray.

We also validated the FCI2 numerical simulations. The experimental results are in good agreement for the timing and the shock velocity, and we have confirmed the simulation procedure with the SESAME 7592 equation of state.

This experimental setup will be used to study the LMJ ablator (CH doped with germanium) on the LIL.

\section{References}

[1] T.R. Boehly, D. Munro, P.M. Celliers, R.E. Olson, D.G. Hicks, V.N. Goncharov, G.W. Collins, H.F. Robey, S.X. Hu, J.A. Morozas, T.C. Sangster, O.L. Landen, D.D. Meyerhofer, Phys. Plasmas 16, 056302 (2009)

[2] J.L. Bourgade, B. Villette, J.L. Bocher, J.Y. Boutin, S. Chiche, N. Dague, D. Gontier, J.P. Jadaud, B. Savale, R. Wrobel, Rev. Sci. Instrum. 72, 1173 (2001)

[3] L.M. Barker, R.E. Hollenbach, J. Appl. Phys. 43, 4669 (1972)

[4] P.M. Celliers, D.K. Bradley, G.W. Collins, D.G. Hicks, T.R. Boehly, W.J. Armstrong, Rev. Sci. Instrum. 75, 4916 (2004)

[5] P. Combis, L. Videau, Internal Report, CEA/DAM (2010)

[6] M. Busquet, Phys Fluids. B. 5, 4191 (1993)

[7] S.P. Lyon, J.D. Johnson, Technical Report LA-UR-92-3407, LANL (1992) 